\title{
La pédagogie de la conscientisation et de l'engagement : pour une éducation à la citoyenneté démocratique dans une perspective planétaire Première partie
}

\section{Pedagogy for Critical Consciousness and Engagement: Education for a Democratic Citizenship in a Global Perspective}

\section{First Part}

\section{La pedagogía de la conscientización y del compromiso : una educación para la ciudadanía democrática en una perspectiva planetaria Primera parte}

Catalina Ferrer et Réal Allard

Volume 30, numéro 2, automne 2002

La pédagogie actualisante : un projet éducatif

URI : https://id.erudit.org/iderudit/1079527ar

DOI : https://doi.org/10.7202/1079527ar

Aller au sommaire du numéro

Éditeur(s)

Association canadienne d'éducation de langue française

ISSN

0849-1089 (imprimé)

1916-8659 (numérique)

Découvrir la revue

Citer cet article

Ferrer, C. \& Allard, R. (2002). La pédagogie de la conscientisation et de l'engagement : pour une éducation à la citoyenneté démocratique dans une perspective planétaire : première partie. Éducation et francophonie, 30(2), 66-95. https://doi.org/10.7202/1079527ar
Résumé de l'article

Cet article comporte deux parties interdépendantes. Dans l'introduction à la première partie, l'éducation à la citoyenneté démocratique dans une perspective planétaire (ÉCDPP) est définie comme cadre de la pédagogie de la conscientisation et de l'engagement (PCE). Un portrait synthèse des défis multiples et complexes posés par la modernité sur les plans humain, social et environnemental est présenté avec, en contrepartie, un portrait des richesses ou des forces qui s'activent pour relever ces défis. Une réflexion sur l'importance d'une ÉCDPP sert d'introduction à une analyse des facteurs qui font que l'éducation mène souvent à des types de conscience qui ne permettraient pas de résoudre les problèmes contemporains et à une réflexion sur l'importance d'une éducation qui conduirait à une conscience ou littératie critique ainsi qu'à un sens éthique de responsabilité à l'égard de ces problèmes. La deuxième partie porte sur la définition et la description de la pédagogie de la conscientisation et de l'engagement proprement dite. Les termes " conscientisation ", « engagement " et « PCE » y sont définis ainsi que la transversalité des objectifs et les critères de classification des contenus de conscientisation abordés. Suivent une description des caractéristiques de la PCE et une analyse des obstacles à la conscientisation critique. En guise de synthèse, sont mis en relief les limites et les risques de la PCE ainsi que ses apports potentiels à la personne et à la société.
Tous droits réservés (C) Association canadienne d'éducation de langue française, 2002
Cecument est protégé par la loi sur le droit d'auteur. L'utilisation des services d'Érudit (y compris la reproduction) est assujettie à sa politique d'utilisation que vous pouvez consulter en ligne.

https://apropos.erudit.org/fr/usagers/politique-dutilisation/ 


\section{La pédagogie de la}

conscientisation et de l'engagement : pour une éducation à la citoyenneté démocratique dans une perspective planétaire

Première partie - Portrait de la réalité sociale et importance d'une éducation à la conscientisation critique et à l'engagement

\section{Catalina FERRER}

Faculté des sciences de l'éducation, Université de Moncton, (Nouveau-Brunswick), Canada

Réal ALLARD

Faculté des sciences de l'éducation, Université de Moncton, (Nouveau-Brunswick), Canada

\section{RÉSUMÉ}

Cet article comporte deux parties interdépendantes. Dans l'introduction à la première partie, l'éducation à la citoyenneté démocratique dans une perspective planétaire (ÉCDPP) est définie comme cadre de la pédagogie de la conscientisation 
et de l'engagement (PCE). Un portrait synthèse des défis multiples et complexes posés par la modernité sur les plans humain, social et environnemental est présenté avec, en contrepartie, un portrait des richesses ou des forces qui s'activent pour relever ces défis. Une réflexion sur l'importance d'une ÉCDPP sert d'introduction à une analyse des facteurs qui font que l'éducation mène souvent à des types de conscience qui ne permettraient pas de résoudre les problèmes contemporains et à une réflexion sur l'importance d'une éducation qui conduirait à une conscience ou littératie critique ainsi qu'à un sens éthique de responsabilité à l'égard de ces problèmes.

La deuxième partie porte sur la définition et la description de la pédagogie de la conscientisation et de l'engagement proprement dite. Les termes " conscientisation ", " engagement " et "PCE " y sont définis ainsi que la transversalité des objectifs et les critères de classification des contenus de conscientisation abordés. Suivent une description des caractéristiques de la PCE et une analyse des obstacles à la conscientisation critique. En guise de synthèse, sont mis en relief les limites et les risques de la PCE ainsi que ses apports potentiels à la personne et à la société.

\section{ABSTRACT}

\section{Pedagogy for Critical Consciousness and Engagement : Education for a Democratic Citizenship in a Global Perspective}

This article is composed of two interdependent parts. In the introduction to the first part, education for democratic citizenship in a global perspective (EDCGP) is defined as the framework of a pedagogy for critical consciousness and engagement (PCE). A synthesis of the multiple and complex problems of the modern world on human, social and environmental scales is presented with, in counterpart, a portrait of the forces that are active in meeting these challenges. A reflection on the importance of EDCGP serves as an introduction to an analysis of factors often causing education to lead to the kinds of consciousness that would not contribute to the solving of contemporary problems, and to a reflection on the importance of education leading to critical consciousness or « literacy » and an ethical sense of responsibility in relation to these problems.

The second part defines and describes the pedagogy for critical consciousness and engagement. The terms, 'critical consciousness', 'engagement' and 'PCE' are defined, and the transversality of objectives and classification criteria of critical consciousness contents are explained. There is then a description of PCE characteristics and an analysis of obstacles to critical consciousness. By way of synthesis, limits and risks of the PCE are analysed and a description is provided of the potential contributions of this pedagogy to the transformation of the person and of society. 


\section{RESUMEN}

\section{La pedagogía de la conscientización y del compromiso : una educación para la ciudadanía democrática en una perspectiva planetaria}

Este artículo está integrado por dos partes interdependientes. En la introducción de la primera parte, la educación para la ciudadanía democrática en una perspectiva planetaria (ECDPP) se define en tanto que marco de la pedagogía de la conscientización y del compromiso (PCC). Se presenta una síntesis de los múltiples retos y complejidades que provoca la modernidad a nivel humano, social y ecológico, y en contraparte se describen las riquezas y fuerzas sociales que entran en acción como respuesta a dichos retos. Una reflexión sobre la importancia de una ECDPP sirve de introducción al análisis de los factores que provocan que la educación haga surgir tipos de consciencia que no permiten resolver los problemas contemporáneos así como una reflexión sobre la importancia de una educación que provocaría el surgimiento de una consciencia o literacia crítica y de un sentido ético de la responsabilidad ante dichos problemas.

La segunda parte aborda la definición y la descripción de la pedagogía de la conscientización y del compromiso propriamente dichos. Los términos « conscientización ", « compromiso " y « PCC » se definen asi como la transversalidad de los objetivos y los criterios de clasificación de los contenidos abordados por la PCC. A manera de síntesis se analizan los límites y los riesgos de dicha pedagogía y se describen sus aportes potenciales a la transformación de la persona y de la sociedad.

Lorsque nous sommes seuls à rêver, cela ne demeure qu'un rêve. Lorsque nous rêvons ensemble, cela n'est plus seulement un rêve, c'est le début de la réalité.

(Dom Helder Camara, archevêque du Brésil)

\section{Introduction}

Appréciation de la vie, respect de la dignité humaine, épanouissement personnel, autonomie, liberté, conscience critique, reconnaissance d'autrui, empathie, coopération, ouverture à la diversité, paix, justice, égalité, solidarité, respect des droits humains, sens de la démocratie pluraliste et de la coresponsabilité sociale, respect de la nature, telles sont les valeurs qui sous-tendent la pédagogie de la conscientisation et de l'engagement, objet du présent article. Cette pédagogie, qui s'inscrit dans le paradigme émergent de l'éducation à la citoyenneté démocratique dans une perspective planétaire (ÉCDPP), représente la méthode par laquelle l'ÉCDPP vise à 
contribuer au développement d'une citoyenneté démocratique capable de répondre aux défis complexes du monde actuel.

L'ÉCDPP est une approche en pleine gestation quant à ses fondements et à sa mise en pratique dans divers contextes socioculturels. Elle cherche à intégrer les courants éducatifs connexes qui se sont développés tout particulièrement dans la deuxième moitié du $\mathrm{XX}^{\mathrm{e}}$ siècle en tant que réponse aux défis de la modernité (Duerr (2000), Ferrer (1997)). Il s'agit de l'éducation pour les droits humains, la paix et la démocratie, de l'éducation pour l'égalité entre les sexes, de l'éducation interculturelle, de l'éducation pour la protection de l'environnement ainsi que de l'éducation au développement et à la solidarité locale et internationale. Ces divers courants ou "regards émergents" sont interdépendants tant sur le plan de l'utopie qu'ils partagent que des valeurs qui les animent. Leur intégration sous le mouvement rassembleur de l'ÉCDPP permet de mieux tenir compte des rapports qui les lient au double regard de la théorie et de la mise en pratique.

Plusieurs éléments de cette démarche pédagogique contemporaine ont été explorés à divers moments de l'histoire depuis l'Antiquité grecque: pensons au dialogue pratiqué par Socrate. Plus près de nous, on trouve ces préoccupations dans les diverses approches à caractère humaniste élaborées au $\mathrm{XX}^{\mathrm{e}}$ siècle qui ont proposé la transformation de l'école pour la mettre au service de l'épanouissement de l'individu et de l'humanisation de la société.

Le concept de citoyenneté étant récent, multiforme et en constante évolution, il s'enrichit de conceptions différentes qui suscitent des débats (Audigier (2000), Pagé (2001)). Nous nous limiterons ici au concept de «citoyenneté démocratique dans une perspective planétaire» que nous avons privilégié dans les projets que nous réalisons dans les provinces atlantiques (Vienneau (2001)) et de concert avec un regroupement d'universités francophones du Québec et du Nouveau-Brunswick (Ferrer (1994)); Lessard, Desroches et Ferrer (1997)). Cette nouvelle conception est elle aussi objet de débats, notamment en ce qui a trait de savoir s'il est pertinent de situer la citoyenneté dans une perspective planétaire puisque la citoyenneté se définit d'ordinaire dans son sens usuel de ce qui a rapport à un territoire ou à un État.

L'ÉCDPP, telle que nous la concevons, cherche à construire une citoyenneté démocratique locale et ouverte au monde. Une citoyenneté qui tienne compte des interdépendances multiples et complexes entre les divers problèmes actuels de l'humanité et solidaire dans la recherche collective d'une nouvelle façon de penser le monde et d'agir comme agents de changement dans la construction d'un monde meilleur.

Dans sa qualité sociale attribut de la démocratie - régime politique organisé sous la base des droits et des responsabilités - cette citoyenneté doit se construire dans le quotidien, dans un espace démocratique où les personnes sont titulaires de droits et assument les responsabilités afférentes (Osorio (1999)). Elle ne peut toutefois se limiter à une vision locale de la réalité sociale. Le monde étant un, les actions locales ont souvent une répercussion mondiale insoupçonnée. Ce qui signifie que nous avons des responsabilités communes comme citoyens de la planète : mes droits sur le plan local peuvent-ils constituer la négation des droits de citoyens ailleurs? 
Disons avec Audigier (2001) que «se réunir sous l'emblème d'une perspective planétaire, c'est affirmer que rien de ce qui arrive aux autres ailleurs ne saurait nous laisser étranger ».

Il va de soi que ce concept large comporte des risques, dont celui de la dilution dans un ensemble aussi vague que plein de bonnes intentions (Audigier (2001)). On ne saurait trop insister sur l'importance de porter un regard critique sur cette nouvelle conception de la citoyenneté afin d'éviter de la transformer en une idéologie dogmatique (Sauvé (1997)).

Pour les besoins du présent article, nous adoptons la définition suivante de l'ÉCDPP :

L'éducation à la citoyenneté démocratique dans une perspective planétaire est une éducation qui tient compte de la réalité entière du monde d'aujourd'hui et de demain. C'est une éducation qui permet à l'élève et à l'enseignant d'acquérir des compétences et de développer des attitudes pour qu'ils puissent exercer, aujourd'hui et pour demain, une citoyenneté consciente, critique, active, responsable et solidaire, afin de favoriser davantage l'émergence d'une démocratie participative dans un cadre social caractérisé par le pluralisme et les interdépendances locales et globales dans des rapports de coopération plutôt que de compétition [...] (CEICI (1998, p. 4))

Une telle conception de la citoyenneté démocratique fait appel à une pédagogie de la conscientisation et de l'engagement.

Sur la scène locale, les valeurs qui sous-tendent la pédagogie de la conscientisation et de l'engagement dans le cadre de la citoyenneté démocratique dans une perspective planétaire, ci-après la PCE, animent la mission adoptée par le ministère de l'Éducation du Nouveau-Brunswick ${ }^{1}$ et par la Faculté des sciences de l'éducation de l’Université de Moncton qui a conçu et adopté la pédagogie actualisante comme cadre conceptuel pour ses programmes de formation initiale en enseignement.

La pédagogie actualisante comporte huit volets qui la définissent, dont celui de la pédagogie de la conscientisation et de l'engagement. Cette double appellation signale on ne peut plus clairement que, si on cherche à transformer la société et l'éducation en vue de construire une citoyenneté démocratique, il importe de favoriser la réflexion critique tout autant que l'engagement réfléchi dans l'action quotidienne.

Le présent article expose la pédagogie de la conscientisation et de l'engagement dans le cadre de l'ÉCDPP telle qu'elle est mise en application à l'heure actuelle à la

1. Par exemple, dans sa mission de l'éducation publique, le ministère de l'Éducation du Nouveau-Brunswick affirme que : " [...] le but de l'éducation publique de langue française est de favoriser le développement de personnes autonomes, créatrices et épanouies, compétentes dans leur langue, fières de leur culture, sûres de leur identité et désireuses de poursuivre leur éducation pendant toute leur vie. Elles sont ainsi prêtes à jouer leur rôle de citoyennes et citoyens libres et responsables, capables de coopérer avec d'autres dans la construction d'une société juste intégrée dans un projet de paix mondiale fondée sur le respect des droits humains et de l'environnement. » (1993, p. 2)

La Faculté des sciences de l'éducation de l'Université de Moncton affirme, pour sa part : « Par ses activités de formation, de recherche, de développement et de service à la collectivité, la Faculté a la mission de contribuer à la défense et à la promotion de la langue française, à l'excellence des interventions éducatives, à l'approfondissement des connaissances en éducation et à la réalisation d'un projet de société visant un monde de paix, de justice et de solidarité ». (FSÉ (1997, p. 4-5)). 
Faculté, principalement dans le cadre de deux cours de la formation initiale : depuis une dizaine d'années dans le cours à option Éducation pour les droits humains et la paix et depuis cinq ans dans le cours obligatoire Fondements de l'éducation dans une perspective planétaire, offert en $5^{\mathrm{e}}$ année. Au niveau des études supérieures, elle est au cœur du cours de maîtrise Séminaire en éducation dans une perspective planétaire. Il est à noter que depuis trois ans, la Faculté accorde une importance particulière à la question de l'éducation en milieu minoritaire. C'est ainsi que nous expérimentons cette pédagogie dans le cadre de deux cours du programme de doctorat en éducation en milieu minoritaire qui a accueilli ses premiers étudiants en septembre 2000, de même que dans le cours Éducation en milieu minoritaire offert pour la première fois en 2001 au programme de baccalauréat en éducation.

L'article comporte deux parties qui, tout en étant interdépendantes, peuvent être étudiées séparément au besoin. La première porte sur la réalité sociale et l'importance d'une éducation à la conscientisation et à l'engagement dans le cadre d'une éducation à la citoyenneté démocratique dans une perspective planétaire. Nous présentons, en premier lieu, un portrait de la réalité sociale et, en deuxième lieu, une réflexion sur l'importance pour la formation initiale d'éduquer à la compréhension critique de la réalité sociale et à l'éthique de la responsabilité. La deuxième traite de la PCE proprement dite. Elle aborde les définitions des concepts, la transversalité des objectifs, les critères de classification des contenus qui intéresse la PCE, les caractéristiques pédagogiques propres à favoriser la conscientisation critique et le sens de l'engagement, les obstacles à la conscientisation critique. En guise de synthèse, nous mettons en relief les limites et les difficultés de la PCE ainsi que ses apports potentiels à la personne et à la société.

Une précision d'ordre général s'impose d'entrée de jeu. Les termes «apprenant " et "apprenante " désignent ici aussi bien les étudiantes et les étudiants qui sont en formation initiale à l'enseignement à qui s'adresse ce collectif que les élèves des écoles avec lesquels ils et elles seront appelés à travailler. Dans un sens, nous désignons également les professeures et les professeurs à l'université ainsi que les enseignantes et les enseignants qui sont dans les écoles ou aux études. Nous postulons, en effet, que toute personne qui entend en encadrer ou en accompagner une autre dans un processus de conscientisation et d'engagement se doit aussi d'être engagée dans son propre processus de conscientisation et d'engagement. Freire (1980) illustre bien ce point de vue lorsqu'il affirme :

L'éducateur n'est plus celui qui simplement éduque, mais celui qui, en même temps qu'il éduque, est éduqué dans le dialogue avec l'élève. Ce dernier en même temps qu'il est éduqué est aussi éducateur. Tous deux deviennent sujets dans le processus où ils progressent ensemble. (p. 62)

C'est dans cet esprit que le présent article invite à faire de notre Faculté, ainsi que de tout lieu d'éducation, un forum de débats, de dialogue pluraliste et interculturel, de clarification d'idées et de valeurs, d'apprentissage de la compréhension critique et de la solidarité. 
La démarche est difficile et, comme toute pédagogie, elle est assujettie à des limites. Nous ne présentons ici ni un modèle achevé, ni des vérités absolues, mais le résultat de nos réflexions et de notre expérimentation à ce stade de notre cheminement. Nous ne saurions non plus prétendre qu'il peut être appliqué à toutes les réalités; le dialogue avec des personnes vivant d'autres réalités socioculturelles que la nôtre revêt ici une importance capitale. Bref, nous nous interrogeons sur cette approche engagée avec le sort de l'humanité et nous cherchons à mieux comprendre sa complexité en collaboration avec les étudiantes et les étudiants ainsi qu'avec nos collègues, animés d'une même conviction : celle de travailler pour une cause sociale juste et porteuse d'espoir en compagnie des humanistes de la planète.

\section{Portrait de la réalité sociale et importance de la PCE}

Il est question ici d'un portrait synthèse de la réalité sociale qui constitue un objet de conscientisation et d'engagement. Cette vision globale de la réalité permet de dégager l'importance d'éduquer à la compréhension critique de la réalité sociale ainsi qu'à l'éthique de la responsabilité.

Ce portrait, que nous avons élaboré comme outil de réflexion pour les besoins de nos cours à la Faculté, présente deux faces de la réalité sociale locale et mondiale : les principaux problèmes sociaux et les principales richesses sociales. Il s'agit d'une vision globale que les médias d'information de masse ne fournissent pas ou très peu et que les étudiantes et les étudiants de la Faculté disent ne pas posséder. C'est avec l'espoir qu'il puisse favoriser, chez nos lectrices et nos lecteurs, la réflexion critique sur la complexité de la réalité sociale et sur le rôle à assumer en tant que citoyens et citoyennes responsables que nous le présentons ici.

Le rationnel pédagogique à la base de l'élaboration de cet outil de réflexion s'appuie sur trois hypothèses de travail.

Premièrement, nous formulons l'hypothèse qu'étudier la réalité sociale en se penchant sur un ensemble représentatif des problèmes sociaux permet d'éviter les risques d'une étude réductionniste centrée uniquement sur l'une ou l'autre des catégories de problèmes, tels la pauvreté, la guerre, le racisme, l'exploitation de la femme et des enfants, le sous-développement ou la destruction de l'environnement, abordée séparément sans tenir compte des liens qui les unissent entre elles. Nous estimons que procéder à partir d'une vision globale des problèmes sociaux dans les différentes sphères de l'activité humaine favorise l'établissement de liens entre eux et, par conséquent, l'approfondissement de leur connaissances ainsi que de la compréhension du contexte dans lequel ces problématiques se développent.

Cependant, cette façon de procéder peut faire naître certains effets pervers (non voulus). D'abord, se limiter à l'étude des problèmes sociaux, même s'ils sont abordés dans une perspective globale, comporte le risque d'une compréhension incomplète de la réalité sociale parce qu'on ne tient pas compte des richesses ou des forces qui cherchent précisément à servir de contrepoids à ces problèmes. Aussi, une prise de conscience critique de la globalité et de l'ampleur inquiétante des problèmes 
mondiaux de l'heure peut-elle faire surgir des sentiments d'impuissance, de peur et de découragement.

C'est pourquoi, en relation avec l'analyse précédente, nous avançons comme deuxième hypothèse qu'étudier la réalité sociale en mettant l'accent autant sur les richesses que sur les problèmes peut contribuer à prévenir la présence des effets pervers signalés. L'étude des richesses sociales et des figures de proue peut, en outre, être porteur d'espoir et fournir des modèles qui favorisent la réflexion et l'engagement social.

Notre troisième hypothèse est la suivante : aborder l'étude de la réalité sociale (problèmes et richesses) à partir de réalités locales proches du vécu des étudiantes et des étudiants peut favoriser la compréhension de réalités qui sont signifiantes pour eux et pour elles et mieux les préparer à comprendre ensuite des réalités plus lointaines. Nous estimons que ces problèmes, quoique près de leur vécu, sont peu ou mal compris, et certains, comme la violence faite aux femmes et aux enfants ainsi que les causes profondes de la pauvreté, sont cachés ou " invisibilisés " par la société locale, comme ils le sont d'ailleurs sur le plan mondial. À ce titre, les problèmes à caractère local devraient, à notre avis, être étudiés dans le cadre de la formation initiale à l'enseignement et dans le contexte scolaire afin de permettre aux apprenantes et aux apprenants d'améliorer leur compréhension des réalités qui sont signifiantes pour eux et pour elles et de repenser leur rôle de citoyens et de citoyennes à leur sujet.

Par ailleurs, parce que ces problèmes touchent de près au vécu, il est à espérer que leur étude sera une source de motivation à l'engagement dans la communauté tout en établissant des liens avec la problématique mondiale.

Enfin, par rapport à cette compréhension de la réalité locale, l'étude des richesses à caractère local et des figures de proue de la communauté peut renforcer cette réflexion en fournissant des modèles puissants parce que ces derniers sont familiers aux apprenantes et aux apprenants.

Les évaluations faites de cette approche par nos étudiantes et nos étudiants confirment, année après année, la pertinence de nos hypothèses de travail.

\section{Portrait de la réalité sociale en ce début du $\mathrm{XXI}^{\mathbf{e}}$ siècle}

La modernité se caractérise autant par sa grandeur que par sa misère. Seul un point de vue qui embrasse l'une et l'autre pourrait nous donner cette vision juste de notre époque dont nous avons besoin pour relever ses plus grands défis. (Charles Taylor (1992, p. 150))

Le $\mathrm{XX}^{\mathrm{e}}$ siècle a été accueilli dans l'enthousiasme et dans la quasi-certitude d'un futur emballant pour toute l'humanité. Cependant, le constat des nombreuses injustices, des guerres et des désastres écologiques de ce siècle, et du désespoir qu'ils ont engendré, a contribué à la prise de conscience de la situation critique dans laquelle se trouve l'humanité. Voilà une partie incontournable de l'héritage du $\mathrm{XX}^{\mathrm{e}}$ siècle et les raisons qui font que l'arrivée $\mathrm{du} \mathrm{XXI}^{\mathrm{e}}$ siècle s'est faite sans enthousiasme et même dans une inquiétude généralisée. 
Selon l'image de Semprún (1999), le XX $\mathrm{X}^{\mathrm{e}}$ siècle est une période de grandes intolérances (l'intolérance ayant toujours existée), mais est aussi celle des droits humains, de la libération de la femme, de la promotion de la démocratie. En effet, le siècle dernier, tout en accomplissant des progrès inouïs dans de nombreux domaines, a été marqué par des problèmes dont nous ressentons toujours les conséquences. Il nous a laissé un double héritage : héritage de mort et d'espérance, dit Morin (2000). Ou de problèmes et de richesses, ajouterions-nous.

\section{Vue d'ensemble des problèmes contemporains}

Les problèmes humains et sociaux hérités du siècle dernier, souvent reliés, sont présents partout, tant à l'échelle locale et régionale qu'à l'échelle nationale et internationale.

Sur le plan intrapersonnel, on observe, entre autres, la montée de l'individualisme ainsi que l'aliénation chez plusieurs, dont les manifestations peuvent prendre la forme de l'autodestruction (toxicomanie, suicide...), de la prédominance du mode " avoir » d'une existence caractérisée, selon Fromm (1978), par le désir passionné de l'argent, de la consommation, de la célébrité, de la puissance et, enfin, de la passivité, qui constitue, selon lui, l'un des traits pathologiques les plus caractéristiques de la société moderne. L'individu se sent seul, impuissant, angoissé, et le conformisme semble être l'une des façons qu'il trouve pour échapper à l'angoisse.

Sur le plan interpersonnel, on est témoin de la violence qui marque une grande partie des rapports interpersonnels au sein de la famille, du milieu de travail et de l'école. Malgré les progrès réalisés en matière de communication humaine, l'incompréhension demeure générale, souligne Morin (2000), et, à ce titre, favoriser la compréhension mutuelle doit être l'une des finalités de l'éducation.

Sur le plan social, rappelons, en premier lieu, le problème de la violence armée toujours omniprésent malgré le discours de paix et de désarmement de la fin du XX siècle. Il y a, d'une part, les guerres (rappelons l'extrême violence de deux guerres mondiales, dont les camps d'extermination nazis et les deux bombes nucléaires lancées par les États-Unis sur le Japon) ainsi que la militarisation de la planète, qui se poursuit avec la fabrication d'armes de plus en plus sophistiquées. La menace de la destruction de l'humanité par l'arme nucléaire, qu'on croyait dissipée à la fin de la guerre froide entre les deux superpuissances, s'accroît avec la dissémination de la bombe et les nouveaux conflits armés qui éclatent. Et, d'autre part, il y a le recours au terrorisme sous ses diverses manifestations, dont le terrorisme d'État, pour tenter d'avoir gain de cause dans des conflits dont les raisons sont multiples et complexes.

Nous sommes dans une économie de guerre caractérisée notamment par la dépendance d'importants secteurs d'économies nationales sur la vente d'armes. Mayor (1999), directeur de l'UNESCO, a déploré le fait qu'un monde qui dépense 800000 millions de dollars par année en armes ne puisse trouver l'argent nécessaire pour assurer l'éducation de tous les enfants (somme estimée à 6000 millions par année pour l'an 2000).

En deuxième lieu, rappelons le problème de la violence structurelle qui occasionne la pauvreté grandissante d'une partie considérable de l'humanité (avec tout 
ce que cela comporte de violation des droits civils et politiques, dont la liberté, ainsi que des droits sociaux, dont particulièrement les droits à l'alimentation, à la santé, à l'éducation, au travail, au loisir...), malgré le discours dominant de la valorisation de la démocratie et du respect des droits humains des dernières décennies. Bon nombre de spécialistes dénoncent l'écart qui se creuse entre pays riches et pays pauvres ainsi que l'écart accru entre riches et pauvres à l'intérieur des pays. Partout, une minorité privilégiée du pays s'enrichit, tandis que la majorité s'appauvrit. Cortina (2001) dénonce le fait qu'à l'ère de la mondialisation une grande partie des habitants de la planète vit dans la pauvreté et est sous-alimentée, pendant que les entreprises d'alimentation inventent des produits destinés à la petite minorité qui possède le pouvoir d'achat nécessaire pour consommer "même ce qu'elle ne sait pas qu'elle désire ". Pour sa part, Ziegler (2001), témoin expert de l'ONU pour le droit à l'alimentation, qualifie de " génocide silencieux » le fait que chaque jour il y ait des milliers de personnes qui meurent de faim de par le monde, la plus grande partie d'entre eux étant des enfants, alors que Le Programme d'alimentation mondial montre que les recours existent pour alimenter le double de la population actuelle de la planète. Il ne s'agit pas d'une fatalité, dit-il, mais d'un crime contre l'humanité parce que le monde n'a jamais été aussi riche.

Pour Habachi (1986), «il n’y a pas des pays développés et des pays sousdéveloppés; il y a seulement des pays mal développés. Ceci signifie que partout (entre pays ainsi qu'à l'intérieur de chaque pays), il y a des pathologies du développement : par excès ou par défaut » (p. 62). Nous sommes, dit Touraine (2001), « devant le triomphe du capitalisme le plus extrême ", avec tout ce qu'il génère de violence et d'injustice sociale.

À ce titre, il convient de rappeler que le début du millénaire actuel est fortement marqué par la controverse que suscite le modèle néo-libéral et la mondialisation. D'une part, celle-ci permet un progrès considérable, notamment dans le domaine de l'économie et des communications, mais de l'autre, elle fait naître de nouveaux problèmes et aggrave certains problèmes existants, tels que la pauvreté et la nouvelle forme d'exploitation des travailleurs au nom de la compétitivité et de la mondialisation des marchés. S'ajoutent à ces fléaux le problème crucial de la consommation et du trafic des drogues ainsi que l'immigration clandestine résultant de la misère à laquelle font face bon nombre des pays du Sud.

Chossudovsky (1998) parle de "mondialisation» de la pauvreté, causée, entre autres, par ce qui contribue à la source de l'endettement du Sud. Il affirme : « Alors qu'une part croissante des produits consommés en Europe occidentale et aux ÉtatsUnis proviennent du tiers-monde, l'économie de rente des pays riches s'approprie près de $80 \%$ du revenu global. Ici se situe la source de l'endettement du Sud [...] (p. 35)

Soulignons, en troisième lieu, le problème de la violence faite aux femmes, laquelle est caractérisée principalement par l'inégalité, l'exclusion ainsi que la violence physique et sexuelle. Ce problème à caractère universel qui remonte à de nombreux siècles demeure actuel malgré les efforts de libération déployés par le mouvement féministe au $\mathrm{XX}^{\mathrm{e}}$ siècle. 
Mentionnons, en quatrième lieu, un problème qui découle directement de la misère dans laquelle vit une grande partie de l'humanité et de l'exploitation dont la femme est victime, celui de l'exploitation des enfants dans le monde en dépit du discours dominant de la défense de leurs droits. Pensons principalement au trafic d'enfants destinés à l'exploitation par le travail, la pornographie et la prostitution. Parallèlement à cette exploitation sociale, les enfants, de par leur condition de dépendance vis-à-vis de l'adulte, sont exposés à toutes sortes de mauvais traitements physiques, psychiques et sexuels, souvent de la part de personnes placées en situation d'autorité et dont le devoir primordial est de veiller à leur formation et à leur protection.

En cinquième lieu, réfléchissons à un autre problème directement lié aux précédents, celui de l'inégalité d'accès à l'éducation. Au XX ${ }^{\mathrm{e}}$ siècle, malgré les efforts non négligeables réalisés en matière d'alphabétisation et d'éducation de la population de la planète, une large proportion de celle-ci reste encore en marge de ce droit fondamental qu'est l'éducation, particulièrement dans les pays pauvres. Selon l'UNESCO (2000), la population estimée d'analphabètes âgés de quinze ans et plus sur la planète sera de 847,3 millions dans les pays pauvres, alors qu'il sera de 10,2 millions dans les pays riches en 2005 et presque les deux tiers de ces analphabètes, tant dans les pays riches que dans les pays pauvres, seront des femmes. Il y a donc inégalité d'accès à l'éducation non seulement entre les pays, mais encore entre les sexes.

Mentionnons aussi le problème de la violence socioculturelle exprimée principalement par la discrimination fondée sur la race ou l'ethnie, le sexe et l'orientation sexuelle ainsi que par la montée des nationalismes xénophobes et des intégrismes idéologiques et religieux.

Ajoutons à ce portrait la violence véhiculée par les médias d'information, le cinéma et les jeux vidéos.

Sur le plan environnemental, rappelons la crise sans précédent dans l'histoire à laquelle fait face l'humanité, celle de la destruction de l'environnement caractérisée, entre autres, par la contamination de l'air et de l'eau, les changements climatiques, la disparition de certaines espèces et la destruction des forêts. Reeves (1988) signale que, depuis l'apparition des humains sur la terre, c'est la première fois qu'une espèce vivante détient le pouvoir de l'équilibre biologique de la planète au point de menacer sa propre survie. Dans cet esprit, Dumont (1988) affirme : «Aujourd'hui, nous savons, en effet, avec certitude que la compétitivité forcenée du libéralisme nous amènera vite à la mort de notre écosystème [...]. La nécessaire réduction des écarts riches/pauvres implique évidemment celle de nos gaspillages, que nous baptisons niveaux de vie. » (p. 281)

La Commission mondiale sur l'environnement et le développement (1988) déclare pour sa part, dans son Rapport (communément appelé le rapport Brundtland), que le temps est venu d'unir économie et écologie et d'assurer un développement équitable, respectueux tant des ressources des générations à venir que de l'écologie naturelle.

Ces problèmes rendent plus urgente que jamais la nécessité d'un changement profond de style de vie dans les pays consommateurs. 
Étant par nature interdépendants, les problèmes sociaux et environnementaux de l'heure appellent des solutions globales et solidaires, mais, bien entendu, adaptées aux caractéristiques de chaque région. Le débat pluraliste et planétaire sur le sujet devient un impératif. Nombreux sont les auteurs qui analysent la globalité de ces problèmes et proposent des pistes de solution ${ }^{2}$.

\section{Problèmes à incidences locales}

Sur le plan intrapersonnel, les jeunes sont au stade de la construction d'une identité qui leur est propre. Ils s'interrogent et cherchent à comprendre, à dégager un sens de prescriptions de tout genre qu'ils reçoivent tant à l'école et dans la famille que dans la communauté et les médias d'information. Nos observations dans la région nous permettent d'affirmer que leurs questions demeurent souvent sans réponses satisfaisantes à leurs yeux. En conséquence, elles et ils sont nombreux à ressentir de la frustration à l'égard d'un monde qu'ils perçoivent comme lourd de contradictions. Des jeunes de la région vivent cette situation avec beaucoup d'anxiété, certains ayant recours à la surconsommation d'alcool et de drogues pour tenter de réduire le stress ou pour fuir leur réalité, ce qui les mène très souvent à la pharmacodépendance. Ne trouvant guère de réponses claires ou de solutions véritables à leurs problèmes, certains vivent des dépressions qui les mènent à des tentatives de suicide ou au suicide même. D'aucuns, devenus contrevenants, sont traduits en justice, risquant ainsi de se renfermer dans un cycle d'autodestruction presque inévitable.

Sur le plan interpersonnel, les apprenantes et les apprenants sont témoins de nombreuses situations où des personnes ont recours à la violence verbale et, parfois même, à la violence physique pour régler leurs conflits. Plusieurs ont subi la violence ou ont eux-mêmes eu recours à la violence dans des situations conflictuelles. Et un bon nombre sont conscients du fait que des hommes de leur milieu ont recours à la violence physique et sexuelle à l'endroit des femmes et des enfants (bien que ce phénomène soit, lui aussi, très souvent « invisibilisé »).

Les apprenantes et les apprenants sont témoins ou sont victimes de différentes formes de discrimination fondées notamment sur la couleur, l'ethnie, la religion, le sexe, la langue, les convictions politiques, l'âge ou la condition sociale. Bon nombre sont euxmêmes auteurs de ces pratiques discriminatoires, tout en étant probablement inconscients souvent des origines de leurs comportements ou de leurs conséquences.

2. Pour des études plus approfondies sur ces questions, se reporter, entre autres, aux sources suivantes : Mayor (1999), sur la violence armée et les guerres; Chomsky (1995) et Chossudovsky (1998), sur la mondialisation et les écarts entre les riches et les pauvres à l'échelle mondiale; Jacquard (1995), sur les risques liés au fondamentalisme du modèle économique dominant; la Commission mondiale sur l'environnement et le développement (1988), sur les problèmes reliés à l'exploitation abusive et à la destruction de l'environnement; Menchú (1985) et Chungara (1981), sur le génocide des Amérindiens; de Beauvoir (1949) et Saint-Jean (1983), sur la discrimination à l'égard des femmes dans I'histoire; Miller (1984) et les rapports annuels de I'UNICEF (2000) par exemple, sur la violence faite aux enfants; Freund (1991) et Breton (1997), sur la mésinformation dans les médias d'information; Mattelart (1992), sur les risques de la monoculture; Fromm (1941), sur la peur de la liberté et sur la tendance de l'individu moderne à vouloir posséder plutôt qu'à vouloir être (1978); Caouette (1997), sur les problèmes humains liés à l'école; Magendzo et Donoso (2000), sur les violations des droits humains dans le système éducatif; Touraine (1997) sur les défis de la démocratie; Thériault (1999) sur l'état des lieux des communautés francophones minoritaires au Canada. 
À l'école, les jeunes se rendent compte qu'on cherche souvent à faire taire ceux parmi eux qui dérangent par leurs questions et leurs critiques de l'ordre établi. Même si les données relatives à l'abandon scolaire au Nouveau-Brunswick sont moins inquiétantes que celles constatées dans d'autres provinces, nos observations nous permettent d'avancer l'hypothèse qu'ils sont nombreux à décrocher psychologiquement de l'école parce qu'ils et elles ont perdu le goût de l'étude, le goût de l'apprentissage et de la recherche et, surtout, la confiance en eux-mêmes ou en ellesmêmes, en leurs ressources et en leur avenir. Les propos de Caouette (1977) à propos de l'école québécoise portent à réflexion :

Il est urgent que cesse un tel gaspillage des ressources humaines et qu'on arrête de déformer, d'aliéner et de déposséder des générations entières de jeunes de leurs véritables ressources et de leurs talents, de leur créativité, de leur émotivité et de leurs rêves, par une école qui cherche à les standardiser et à les uniformiser, à les mettre au pas et en rangs pour éliminer progressivement les moins dociles. (p. 37)

Sur le plan social, dans le domaine socioéconomique, les apprenantes et les apprenants habitent une région où des familles et des enfants sont aux prises avec la pauvreté; certains vivent même cette situation. Des travailleurs et des travailleuses sont menacés de perdre leur emploi, d'autres sont en chômage, et, dans de nombreux secteurs, les femmes reçoivent un salaire inférieur à celui des hommes pour un travail similaire.

Dans le domaine sociopolitique, les gens des provinces atlantiques ont été exposés (à tout le moins par les médias) aux affrontements entre groupes autochtones et blancs au sujet des droits de pêche et de coupe de bois, qui font partie des revendications historiques des communautés autochtones de la région. La couverture médiatique de tels événements leur a permis d'observer la violence qui caractérise ces conflits, mais ne leur a pas fourni en même temps des éléments d'analyse approfondie des enjeux sociohistoriques, socioéconomiques, psychologiques et environnementaux des conflits.

Dans le champ socioculturel et linguistique, les apprenantes et les apprenants francophones sont soumis aux influences des inforoutes et des médias de masse contrôlés ou dominés par de puissantes multinationales. Celles-ci contribuent à l'hégémonie et à l'impérialisme de la langue anglaise et de la culture des États-Unis qui mènent à l'affaiblissement et, inévitablement peut-être, à la disparition de leur langue maternelle et de leur culture. En effet, plusieurs s'assimilent linguistiquement et culturellement, souvent sans avoir conscience des enjeux que présente leur assimilation pour eux ou pour la communauté. Par ailleurs, ils et elles sont témoins des tensions qui divisent les communautés linguistiques anglophone et francophone et des luttes menées par la communauté francophone pour préserver sa langue et sa culture (Allard (2002), Landry et Allard (1997)). Quelques-uns peuvent savoir que les communautés autochtones luttent également contre l'assimilation et l'acculturation (phénomène qui est souvent « invisibilisé " par les médias régionaux). (Bear-Nicholas (1997)). 
Sur le plan environnemental, les apprenantes et les apprenants ont été exposés à l'inquiétude de plus en plus grande exprimée par les écologistes qui font état des nombreux problèmes liés à la « mal exploitation » de l'environnement dans la région. Parmi ces problèmes, mentionnons la surexploitation et la dévastation de forêts entières qui entraînent des effets néfastes sur les plans humain et écologique, la surpêche de certaines espèces de poissons et de crustacés et ses conséquences désastreuses tant pour les espèces que pour les familles et les communautés qui en dépendaient économiquement. Il ne faut pas oublier, enfin, les pratiques industrielles et individuelles qui ont contribué à la pollution de l'atmosphère, de la nappe phréatique et des cours d'eau de la région et qui menacent la santé des humains et la survie de certaines espèces animales et végétales.

\section{Vue d'ensemble de richesses contemporaines}

Les richesses ou les forces sociales héritées du siècle dernier, souvent reliées, sont elles aussi, comme le sont les problèmes sociaux, présentes partout, tant à l'échelle locale et régionale qu'à l'échelle nationale et internationale.

Sur le plan intrapersonnel, des accomplissements majeurs ont marqué les dernières décennies grâce à l'action de bon nombre de personnes et de groupes de par le monde à la recherche du sens de la vie. On a vu se développer une vision holistique de la personne, une prise de conscience de l'importance de la qualité de la vie et du respect de la dignité de tout être humain.

Sur le plan interpersonnel, par rapport aux démarches entreprises sur les plan intrapersonnel et social, on a pu observer une évolution significative des rapports entre les sexes et les ethnies vers plus d'égalité et d'appréciation. L'importance de l'ouverture et la reconnaissance de l'autre deviennent matière de réflexion grâce, entre autres, à l'action des courants éducatifs engagés dans l'éducation interculturelle et dans l'éducation pour les droits humains et la paix.

Sur le plan social, des accomplissements majeurs ont marqué le $\mathrm{XX}^{\mathrm{e}}$ siècle grâce à l'action de mouvements sociaux qui se sont créés dans le monde, dont le mouvement de la libération de la femme qui a réalisé, d'après plusieurs spécialistes, la plus grande révolution du siècle (quoiqu'il reste encore un long chemin à parcourir pour que soit assurée l'équité pour toutes les femmes); le mouvement pacifiste et de revendication des droits humains (bien qu'ici aussi un long chemin reste à parcourir, en somme, pour accorder la priorité à la résolution non violente des conflits et pour passer des discours et des déclarations de principe aux actes).

Dans le champ juridique international, il faut souligner la signature par les Nations Unies de trois documents juridiques à caractère universel :

1. en 1948, la Déclaration universelle des droits de l'homme,

2. en 1959, la Déclaration des droits et de l'enfant et,

3. en 1990, la Convention relative aux droits de l'enfant.

Malgré le fait que ces droits restent encore souvent théoriques, ces documents constituent un référent essentiel dans la lutte pour le respect des droits et de la dignité humaine. Saramago (2000) affirme que les droits humains constitueront la 
grande utopie du XXI ${ }^{\mathrm{e}}$ siècle. Pour sa part, l'UNESCO adopte en 2001 la Déclaration universelle sur la diversité qui, selon son directeur, compte désormais parmi les textes fondateurs d'une nouvelle éthique promue par l'UNESCO au début du siècle actuel.

Parmi les accomplissements sociaux, mentionnons la décolonisation de l'Afrique (bien qu'elle ait, en revanche, trop souvent cédé la place à différentes formes de néocolonialisme); la fin de l'Apartheid en Afrique du Sud, même si tout le chemin de libération reste à construire ${ }^{3}$; la décolonisation de l'Asie (dont l'important mouvement de non-violence de Mahatma Gandhi qui a obtenu l'indépendance de l'Inde et inspiré l'Occident); le développement des démocraties (quoique fragiles, inachevées et souvent formelles plutôt que participatives); les luttes ouvrières et la solidarité syndicale menant à des gains appréciables en ce qui concerne les droits des travailleurs et des travailleuses dans de nombreux pays (mais ces gains sont menacés et, dans certains cas, abolis aujourd'hui par la mondialisation).

À titre d'illustration de la vitalité de la société civile, il convient de souligner ici deux faits historiques récents à caractère international : la Marche mondiale des femmes organisée en 2000 partout dans le monde pour revendiquer l'élimination de la pauvreté et de la violence faite aux femmes et aux enfants, et les manifestations des mouvements sociaux en opposition à la mondialisation néo-libérale (Forum continental sur l'éducation (2001)). Organisées en contrepoids aux sommets des grandes puissances, leurs manifestations ont attiré l'attention des médias, ce qui a permis de faire connaître leurs revendications d'une société plus humaine, juste, démocratique et solidaire dans la recherche de solutions.

Dans le domaine de la science et de la technologie, le $\mathrm{XX}^{\mathrm{e}}$ siècle a connu un avancement prodigieux, notamment dans les domaines de la santé et des communications (bien que les pays pauvres voient s'agrandir le fossé qui les sépare des pays riches en ce qui a trait notamment à la santé et aux communications, compte tenu de leurs coûts énormes). Ce progrès scientifique inégal pose également de sérieux problèmes d'ordre éthique et rend manifeste la nécessité de le contrôler, notamment dans les domaines des armes, du clonage humain et des modifications génétiques.

Dans le champ des arts et de la littérature, il va sans dire que le $\mathrm{XX}^{\mathrm{e}}$ siècle a été d'une très grande richesse. Les arts, grâce à l'avancement de la technologie, sont, en effet, plus accessibles au grand public, notamment dans le cas de la musique, de la littérature et du cinéma (mais, hélas, une très grande partie de la population mondiale ne participe pas à cette évolution).

Soulignons, par ailleurs, le rôle d' « éveilleurs » de conscience qu'ont joué et que continuent de jouer les artistes au sein de la communauté, ainsi que l'engagement explicite de nombreux artistes dans les causes sociales, dont le groupe d'artistes pour la paix. "De tout temps ", affirme le poète Gérard LeBlanc (2001), " les artistes ont tenté de rendre sensibles, intelligibles, les bonheurs et les malheurs de leur communauté, leurs déceptions et leurs rêves. En d'autres termes, la création sera toujours

3. Comme l'a dit Nelson Mandela (1994) lors de son élection historique à la présidence de son pays : " Nous ne sommes pas encore libres; nous avons simplement acquis la liberté d'être libres, le droit de ne pas être opprimés. Nous n'avons fait que le premier pas de notre voyage sur une route longue et difficile ». 
une main tendue vers l'autre, un appel de partage d'une vision, un pont entre les êtres humains ».

Dans le domaine de l'éducation, c'est depuis la fin du XIX ${ }^{\mathrm{e}}$ siècle que des courants pédagogiques se développent afin de transformer l'école, trop centrée à leurs yeux sur la transmission de la connaissance. Pensons à l'École Nouvelle, à la pédagogie humaniste, à la pédagogie libertaire, au courant transpersonnel, au courant psychanalytique, à la pédagogie progressiste, à la pédagogie de la libération ou de la conscientisation, à la pédagogie institutionnelle, à la pédagogie critique, à la pédagogie ouverte, au constructivisme et au socioconstructivisme.

Parallèlement à ce mouvement du renouveau de l'école, on voit se développer, faut-il le répéter, des courants éducatifs engagés socialement, telles l'éducation aux droits humains et à la paix, l'éducation pour l'égalité des sexes, l'éducation au développement et à la solidarité internationale, l'éducation interculturelle, l'éducation aux médias... Ces courants prennent davantage racine au sein de l'éducation informelle ou populaire qui se développe en vue de favoriser la transformation de l'éducation traditionnelle et de la société capitaliste (Osorio (1988)). C'est ainsi qu'on voit apparaître partout dans le monde de nombreuses organisations non gouvernementales qui se donnent pour mandat d'éduquer la population à une citoyenneté active, critique, démocratique. Plusieurs parmi elles collaborent avec l'école.

Ajoutons à cela la création de l'UNESCO (Organisation des Nations Unies pour l'éducation, la science et la culture) en 1945, qui a donné une portée internationale aux revendications éducatives dans ce domaine. D'ailleurs, l'UNESCO affirme dans son acte constitutif: "Les guerres prenant naissance dans l'esprit des hommes, c'est dans l'esprit des hommes que doivent être élevées les défenses de la paix ». Cohérente avec cette déclaration, elle lance à l'éducation le défi de contribuer au développement d'une culture de paix et de respect des droits humains (UNESCO (1993)).

Sur le plan environnemental, grâce à l'action des écologistes, il s'est développé une conscience de l'ampleur de la crise de l'environnement causée par l'intervention technique de l'être humain. La destruction de l'environnement qui en résulte a permis de prendre conscience de la fragilité de la nature, vulnérabilité, dit Jonas (1997), « qui n'avait jamais été pressentie avant qu'elle ne soit manifestée à travers les dommages déjà causés » (p. 24). On a pris conscience de l'interdépendance planétaire, des liens étroits entre paix, démocratie, développement et protection de l'environnement, ainsi que de la nécessité éthique de changer de paradigme et de mode de vie afin d'assurer la survie de la planète.

\section{Richesses à incidences locales}

Sur le plan intrapersonnel et interpersonnel, on observe dans la région la même évolution des mentalités qui a été signalée au moment de parler des richesses mondiales.

Sur le plan social, des organismes cherchent à promouvoir les relations harmonieuses entre les francophones, les anglophones et les Autochtones. Dans leur lutte contre l'assimilation linguistique et l'acculturation - phénomènes pouvant 
mener à la disparition de langues ou de cultures dans la région de l'Atlantique - deux groupes ethnolinguistiques, les francophones et les Autochtones, interviennent auprès des instances gouvernementales fédérales, provinciales et municipales afin de faire valoir leurs revendications.

En ce qui concerne la communauté acadienne et francophone, son principal objectif est d'assurer sa vitalité actuelle et future en se dotant des institutions et des services de langue française qui lui permettront de se développer culturellement tout en conservant sa langue. Par exemple, après des années de luttes, les communautés acadiennes et francophones de l'Île-du-Prince-Édouard, du Nouveau-Brunswick, de la Nouvelle-Écosse et de Terre-Neuve-et-Labrador se sont dotées d'écoles homogènes de langue française. Les universités de langue française de la région, l'Université de Moncton et l'Université Sainte-Anne, contribuent à la formation de professionnels ainsi que de chefs de file de ces communautés en menant des recherches collaboratives dans le milieu. En 1999, l'Université de Moncton a créé un doctorat en éducation dont le thème est l'éducation en milieu francophone minoritaire. Pour leur part, les artistes de la communauté témoignent d'une très grande créativité et contribuent de façon fort significative à l'identité sociale acadienne et francophone et à la vitalité de leur communauté.

Dans le champ de la justice sociale, des organismes s'engagent en faveur notamment de la défense et de la promotion des droits humains en luttant contre les phénomènes de la pauvreté, de la violence faite aux femmes et aux enfants, du sexisme, de la xénophobie et du racisme.

Sur le plan environnemental, des groupes travaillent à l'établissement de zones protégées en forêt, dans les marais et sur les côtes, au développement durable dans le domaine de la foresterie et des pêches, à la protection de l'environnement au moyen, entre autres, de programmes de recyclage dans les écoles, les universités et les municipalités, et d'activités visant le rétablissement de cours d'eau, comme la rivière Petitcodiac, à Moncton, dans le sud-est du Nouveau-Brunswick.

\section{Importance d'éduquer à la compréhension critique de la réalité sociale et environnementale et au sens éthique de la responsabilité}

L'avenir de l'humanité est la première obligation du comportement collectif humain à l'âge de la civilisation technique devenue " toute-puissante " modo negativo [...] c'est une responsabilité métaphysique en et pour soi, depuis que l'homme est devenu dangereux non seulement pour lui-même, mais pour la biosphère entière. (Hans Jonas (1997, p. 187))

Devant cette situation mondiale paradoxale caractérisée, d'une part, par la présence de forces destructives qui mettent en péril l'avenir de la planète et de l'humanité, et, d'autre part, par la présence de forces constructives porteuses d'espoir, une double nécessité se fait sentir plus que jamais sur le plan éducatif : la première a trait à la nécessité de développer une conscience humaniste planétaire, c'est-à-dire une conscience critique de l'interdépendance planétaire et de l'ensemble des problèmes et des richesses de l'humanité dans l'espace (ici et ailleurs) et dans le temps (hier et 
aujourd'hui) ainsi que des facteurs qui expliquent cet état de choses afin de pouvoir les résoudre. La deuxième a trait à la nécessité éthique d'assumer la responsabilité à l'égard de l'humanité.

C'est le défi que cherche à relever l'ÉCDPP en mettant en pratique une pédagogie de la conscientisation et de l'engagement dans le cadre scolaire et, par voie de conséquence, dans la formation initiale à l'université.

Viser le développement d'une conscience critique, humaniste, à l'échelle planétaire peut paraître, malgré son intérêt, un objectif trop idéaliste, ambitieux ou exagéré, suivant la mise en garde de bon nombre de spécialistes. Mais, pour Weinstein (1988), parmi d'autres, le développement d'une telle conscience est rendu nécessaire par des conditions sociales objectives et réelles, et le travail réalisé dans le monde par des personnes engagées dans la promotion des droits humains montre qu'il est possible pour l'être humain de développer une conscience humaniste et solidaire résolue à assurer la défense de la dignité de tout être humain.

La compréhension de la réalité mondiale constitue un pas important dans la lutte pour renverser la vapeur; la première chose à faire pour réaliser toute espèce de changement du système consiste, dit Chomsky (1986), à reconnaître les formes existantes d'oppression.

Cette reconnaissance des injustices signifie qu'il faut dénoncer le modèle néolibéral de l'économie de marché (Bourdieu (1999)), qui « sous couvert de modernisation, entend refaire le monde en faisant table rase des conquêtes sociales et économiques résultant de cents ans de luttes sociales, et, désormais dépeintes comme autant d'archaïsmes et d'obstacles au nouvel ordre naissant ». Cet auteur affirme que la nécessité de développer, en chaque citoyen et chaque citoyenne, les dispositions internationalistes est désormais une condition préalable à l'établissement des stratégies efficaces de résistance au modèle dominant. L'appel est à la solidarité.

On voit bien que le défi est double : celui d'éveiller les consciences à la réalité sociale locale et mondiale et celui de s'engager pour la changer. Ce défi exige l'éducation à la citoyenneté démocratique de façon à élargir la démocratie à l'ensemble de l'activité sociale en faisant appel à l'organisation de la société civile, à chaque citoyenne et à chaque citoyens, afin de répondre aux problèmes de l'heure.

En somme, l'ampleur et la nature des problèmes sociaux et environnementaux actuels ainsi que leurs conséquences pour la vie sur notre planète sont telles qu'un nouvel impératif éthique mondial s'impose : celui d'assurer, d'une part, les conquêtes sociales et économiques obtenues à travers des longues luttes sociales de par le monde, de les approfondir et de les rendre extensives à l'ensemble de l'humanité, et celui, d'autre part, d'agir dans une perspective prospective de façon à ne pas compromettre la possibilité future d'une vie humaine.

Comme le montre bien Jonas (1997), la responsabilité pour l'avenir entraîne un nouveau rapport dans la praxis éducative : elle doit viser à ce qu'une vie proprement humaine soit toujours possible sur la terre.

On le voit, cette tâche éthique, mais aussi politique au sens large du terme, interpelle l'éducation à l'échelle planétaire : "Il faudrait arriver à ce que les individus se rendent compte qu'ils appartiennent à une communauté mondiale, en 
même temps qu'à une communauté locale et nationale, et, que leurs décisions et leurs modes de vie ne sont pas sans lien avec ceux des autres peuples du monde». (UNESCO (1984, p. 9))

L'agir local avec une pensée planétaire recouvre diverses capacités que l'éducation serait appelée à développer, affirme Osorio (1995) : former des individus qui, en vue d'assumer leurs responsabilités citoyennes, sont capables de comprendre de façon critique ce monde et sont dotés d'une vision holistique et animés d'un sentiment d'appartenance à l'humanité qui leur permettent de transcender la vision locale et d'établir des liens entre le local et l'universel. Des individus capables d'assumer la responsabilité éthique, qui selon Camps (1994), signifie «la capacité de répondre pour les valeurs que nous souhaitons maintenir et propager ». (p. 86)

Disons avec Perrenoud (2000) que « le défi est celui de développer une double citoyenneté : apprendre à se concevoir et à agir comme citoyen de la Terre, sans cesser d'appartenir à des communautés plus restreintes, en restant conscients des interdépendances multiples entre le local et le global.

L'invitation consiste donc à former des sujets, acteurs de leur propre vie et liés à celles des autres citoyens et citoyennes de la planète, à penser globalement et à agir localement.

Il est impossible de savoir si des efforts sérieux et appliqués suffiront pour résoudre les graves problèmes sociaux et environnementaux du début du XXI ${ }^{\mathrm{e}}$ siècle, mais, disons avec Chomsky (1996) : " Nous pouvons être sûrs, cependant, que l'absence de tels efforts signifiera le désastre " (p. 112). À ce sujet, rappelons que Fromm (1983) faisait ce même appel à la responsabilité en pleine guerre froide : « Je crois que les chances de paix sont très minimes. Mais je crois tout autant qu'à partir du moment où la vie d'un individu est en jeu, ainsi que celle de la société, on n'a pas le droit de calculer les chances de paix, ni de se livrer à des pronostics; au contraire, on doit agir et établir des projets tant qu'il existe un espoir de sauver la paix » (p. 170).

Le débat reste ouvert, et l'important du point de vue pédagogique, croyonsnous, est d'inviter les apprenantes et les apprenants à participer à cette réflexion et à élaborer en commun, dans une démarche critique, les valeurs qui guideront la construction de ce projet visant un monde plus humain, juste et solidaire de même que le comment faire dans le cadre scolaire et dans celui de la formation initiale à l'enseignement pour y parvenir collectivement.

Le problème est alors posé de savoir quel type d'éducation est le plus susceptible de favoriser le mieux l'atteinte de ces objectifs. C'est d'ailleurs sur la nature de l'éducation que nous nous tournons dans la prochaine section.

\section{Quelques défis à relever relativement à la conscientisation par l'éducation}

Pour la première fois dans l'histoire, la survie de la race humaine dépend d'un changement radical du cœur humain. Mais ce changement n'est possible que dans la mesure où interviennent des changements économiques et sociaux capables de donner au cœur humain la chance de changer et le courage et l'envie d'accomplir ce changement. (Erich Fromm (1978, p. 26)) 
La réalité sociale appelle un repositionnement du rôle de l'éducation. Dans notre cadre de pensée, l'éducation, qu'elle soit formelle ou informelle, est appelée à contribuer à ce changement du cœur humain dont parle Fromm. Bien que l'éducation ne puisse opérer seule les transformations nécessaires, elle peut, par sa nature, apporter une contribution significative à la formation de personnes épanouies, capables d'établir des rapports de respect mutuel et de coopération, des personnes ouvertes à autrui et au monde et possédant une conscience humaniste planétaire qui les motive à s'engager socialement en tant que citoyennes et citoyens critiques, responsables et solidaires du sort de l'humanité et de la planète.

Il est peut-être utile de rappeler la relation d'influence réciproque qui relie la société et l'école. En tant que système destiné à éduquer les nouvelles générations, l'école doit poursuivre les finalités établies par la communauté qu'elle dessert, mais elle possède néanmoins, dans les sociétés démocratiques, une certaine autonomie qui lui permet de faire des choix différents. Autrement dit, le système éducatif est influencé par le paradigme dominant et, à son tour, il peut contribuer à changer ce paradigme (Bertrand (1998)). C'est à ce degré d'autonomie du système éducatif que fait appel la PCE.

Nombreux sont les phénomènes qui font obstacle, au sein même de l'éducation, au développement de cette conscience et de cet engagement. Examinons ces défis sans pour autant prétendre qu'ils sont les seuls.

\section{Quand peu de scolarisation (ou son absence) contribue à une conscience intransitive}

Nous avons vu plus haut que l'inégalité d'accès à l'éducation sévit encore tant entre les pays qu'entre les sexes. Ces réalités comportent de graves conséquences pour les personnes qui les vivent ainsi que pour les communautés touchées.

Dans l'analyse de ses observations sur le terrain, Freire (1973) fait observer que les personnes qui sont peu scolarisées ou qui n'ont pas eu accès à l'école connaissent souvent plutôt mal les facteurs sociaux qui influent négativement ou positivement sur leur vie, leur communauté et leur société. Il estime qu'une telle situation entraîne très souvent chez ces individus le développement d'une conscience intransitive, c'est-à-dire une conscience magique ou naïve des situations et des phénomènes. On tend alors à croire que les conditions humaines, qu'elles soient difficiles ou bonnes, qu'il s'agisse des siennes ou de celles d'autrui, s'expliquent par le hasard ou par des facteurs qui échappent entièrement à la volonté. Aussi tend-on à se résigner et à accepter sa situation difficile, s'estimant impuissant et incapable de mener des actions destinées à l'améliorer.

\section{La scolarisation : chemin pouvant mener à une conscience semi-transitive}

L'observation des nombreux problèmes liés à une scolarisation déficiente peut nous amener à conclure qu'il suffirait d'augmenter le nombre d'années de scolarisation pour répondre aux défis du nouveau millénaire. Cependant, même dans les pays riches où les structures éducatives sont bien en place, la question se pose de savoir comment il se fait qu'il y ait, d'une part, un taux aussi élevé d'abandon scolaire et, 
d'autre part, autant de jeunes qui ne savent ni écrire ni lire assez bien pour remplir les exigences de la modernité. Par exemple, dans le cadre d'une enquête internationale consacrée à l'alphabétisme et réalisée en 1994, Statistique Canada (1998) a révélé qu'environ la moitié des jeunes de 16 à 25 ans du Nouveau-Brunswick n'avaient pas les connaissances et les capacités requises pour comprendre et utiliser l'information contenue dans des textes destinés au grand public, pour comprendre et utiliser des textes schématiques comme les cartes, les tableaux et les graphiques ou pour appliquer des opérations arithmétiques à des chiffres sur des documents imprimés.

À ce sujet, bon nombre de professeurs et de professeures d'université - du moins en Amérique du Nord - se demandent comment tant d'années d'efforts dirigés vers la scolarisation peuvent donner de si piètres résultats.

À l'instar d'autres pédagogues, Freire (1973), (1980) soutient que le développement des qualités nécessaires pour relever les défis sociaux ne dépend pas seulement du nombre d'années d'études, mais du type d'éducation. Cet auteur estime que l'éducation formelle traditionnelle, étant de caractère « bancaire " (l'adulte dépose le savoir chez l'élève comme on dépose de l'argent à la banque), mène habituellement au développement d'une conscience semi-transitive des phénomènes. Il s'agit d'une conscience plutôt unidimensionnelle des phénomènes, caractérisée par une pensée à court terme, qui ne prend pas en considération la globalité de la situation et du contexte. Elle mène souvent à des interventions sur des problématiques isolées qui ne tiennent pas compte des liens entre les différents problèmes et leurs causes fondamentales et qui ne visent pas à trouver de solutions à long terme.

En général, les personnes qui développent cette forme de conscience ont le sentiment, souvent illusoire, de comprendre, et le sentiment de pouvoir faire les changements nécessaires pour régler des problèmes sur les plans personnel et social. Mais leur conscience semi-transitive les amène fréquemment, sans qu'elles s'en rendent compte, à régler ces problèmes d'une manière telle qu'elles contribuent à en créer d'autres. Parce qu'elles ne cherchent pas à saisir dans leur globalité les problèmes qu'elles observent dans un contexte donné, ou les enjeux de ces derniers, elles ne sont pas habituellement en mesure de remettre en question le système social qui sous-tend le problème analysé et d'agir en conséquence (Shor (1992)).

Une précision s'impose. Tout au long de ce texte, nous nous efforçons de nuancer nos propos de façon à faire ressortir la complexité du processus éducatif et ses effets possibles chez les personnes. C'est dans cet esprit que nous tenons à souligner que nous ne sommes pas sans savoir que, parmi les personnes qui ont été formées dans l'école « bancaire », certaines ont développé une grande capacité critique et créatrice, échappant au conditionnement de cette école. C'est le cas justement, parmi tant d'autres, des spécialistes qui critiquent l'école "bancaire». Comme le souligne Savater (1998), l'éducation, "à l'égal de tout engagement humain, a des limites et ne réalise jamais qu'une partie de ses meilleures - ou de ses pires - ambitions» (p. 16). Rappelons que les conséquences de l'école sur chaque individu dépendent d'un ensemble de facteurs importants, dont le contexte familial et social. 
Mais poursuivons l'analyse et réfléchissons à quelques phénomènes qui contribuent à renforcer, pour ainsi dire, la conscience semi-transitive qui résulte de l'éducation «bancaire».

La spécialisation dans l'éducation formelle. Si la spécialisation qui caractérise souvent l'enseignement secondaire et celle, parfois extrêmement prononcée, qui caractérise l'enseignement universitaire, apporte des gains quant à l'analyse, elle constitue néanmoins un handicap pour la synthèse. Entre autres, elle ne contribue pas à la prise de conscience globale des phénomènes et de leur contexte, et elle ne permet pas de saisir et d'analyser les interdépendances et les complémentarités entre les spécialités. Par ailleurs, ces conséquences négatives s'aggravent par la rareté de moments et d'espaces réservés à la réflexion critique, phénomène qui afflige nos sociétés modernes.

Les lacunes engendrées par la spécialisation et le manque de temps de réflexion critique ne se traduisent pas uniquement par l'absence de connaissances dans les domaines négligés, mais par la méconnaissance, voire la "malconnaissance ", de données dont la compréhension est essentielle à la vie du citoyen et de la citoyenne.

Une éducation au service des valeurs de la consommation et de la marchandisation. Petrella (2000) montre que, dans le contexte de la mondialisation actuelle, l'éducation est menacée par cinq pièges reliés à l'explosion d'un mode de vie centré sur la consommation des masses et par la marchandisation de l'activité humaine. Une éducation au service de telles valeurs ne peut faire autrement que de contribuer elle aussi au développement d'une conscience semi-transitive. Voyons brièvement ces pièges.

Premier piège : «L'éducation pour la ressource humaine, ou comment l'éducation pour et par la personne humaine a été évincée ». La personne que l'on veut former dans le cadre de la mondialisation n'est plus un sujet social, mais une ressource, au même titre que toute autre marchandise économique. Elle devient une ressource gérée qu'on prépare, transforme, exploite, utilise, recycle et remplace, comme on le fait pour toute ressource, en fonction des besoins de l'entreprise, de sa compétitivité et de sa survie dans la course à la productivité et au profit.

Deuxième piège : «L'éducation non marchande devenue éducation marchande, ou comment l'éducation a été soumise à l'économie capitaliste de marché ». Dans ce modèle, l'éducation est appelée à être un marché au service des grandes entreprises. De plus en plus, ces dernières mènent la danse de l'éducation, soutenues par les pouvoirs publics nationaux convaincus qu'ils doivent favoriser la compétitivité de leurs propres entreprises.

Troisième piège : «L'éducation comme instrument de survie à l'ère de la compétitivité mondiale, ou comment l'éducation a été transformée en un “ lieu ” où l'on apprend une culture de guerre (" mieux réussir que les autres et à leur place ») plutôt qu'une culture de vie (« vivre ensemble avec les autres dans l'intérêt général »).

Quatrième piège : "L'éducation au service de la «technologie », ou comment la "technocratie " s'est emparée du pouvoir de donner sens et orientation à la connaissance et à l'éducation ». Dans le modèle de la mondialisation, ce n'est plus la 
demande sociale qui dicte les besoins en éducation, mais l'outil qui définit le rôle et l'utilité de l'éducation.

Cinquième piège : «L'éducation pour l'égalité cédant le pas à l'éducation pour l'équité, ou comment, dans une société de la connaissance qui considère le savoir comme la principale source de création de richesse, le système éducatif sert à légitimer de nouvelles formes de stratification et de division sociales ». Sur le plan mondial, la logique marchande de l'éducation, la connaissance traitée comme capital économique et la révolution des multimédias sont en voie de créer une économie par laquelle, partout dans le monde, "un fossé de plus en plus profond se creuse entre les " qualifiés " (ceux et celles qui ont accès à la connaissance qui compte) et les “non-qualifiés " (ceux et celles qui n'ont pas eu le droit d'accéder à cette connaissance ou qui l'ont perdue)».

Se libérer de ces pièges constitue une tâche d'importance primordiale qui interpelle l'éducation elle-même ainsi que l'ensemble de la société, et il est possible d'y parvenir, affirme Petrella.

Les médias d'information et l'Internet. D'aucuns pourraient croire que les médias d'information et l'Internet pourraient suppléer l'éducation formelle de caractère bancaire ou combler certaines des carences des approches éducatives qui développent la conscience semi-transitive. Nous ne saurions nier la contribution indéniable des médias, en ce sens qu'ils sont souvent des " chiens de garde ", pour ainsi dire, de la démocratie et des portes d'accès à la culture pour les masses, ainsi que d'Internet, qui a démontré son importance et son potentiel pour la participation aux débats et aux actions visant, par exemple, à contrer des injustices. Par contre, d'une part, il y a inégalité dans l'accès aux médias d'information et à l'Internet. D'autre part, s'il est vrai que les médias informent la population au sujet d'une grande quantité des problèmes de l'humanité, il est aussi vrai qu'ils ne fournissent que très rarement des instruments d'analyse de ces problèmes, ou qu'ils présentent la diversité des actions positives menées par des organismes ou des individus forces vives de la société - visant à les résoudre. Ces deux caractéristiques des médias d'information sont fort susceptibles d'alimenter chez plusieurs un double sentiment de saturation et d'impuissance. Selon Wurman (1989), le fait d'avoir accès à des quantités considérables d'informations sans posséder les outils qui permettraient de les organiser en connaissances signifiantes provoque souvent de l'anxiété.

Il y a lieu de se demander si les effets combinés d'une anxiété provoquée par la difficulté de dégager cohérence et sens de toutes ces informations et d'un sentiment d'impuissance par rapport aux possibilités de mener une action signifiante ne conduiraient pas la personne à un refus plus ou moins global de l'information sur le monde et à un repli égocentrique.

Par ailleurs, la multiplication de l'information qu'on reçoit peut faire croire qu'on est bien renseigné sur les principaux aspects de la réalité mondiale. Cette croyance erronée empêcherait-elle de chercher à mieux s'informer? Par surcroît, on ignore souvent l'existence du processus de filtrage de l'information et les intérêts politiques et économiques qui sont à la base de ce filtrage. Cette situation contribue à l'ignorance généralisée de la puissance de la manipulation des affects et de la 
manipulation cognitive qu'exercent certains médias de masse (Breton (1997); Chomsky (1986), (1996); Freund (1991)). L'ignorance du phénomène de la manipulation n'aurait-elle pas pour effet de ne pas inciter la personne à remettre en question l'information reçue? Surinformée et mal informée à la fois, la personne est limitée à une conscience intransitive ou semi-transitive des phénomènes.

Pour ce qui est de l'influence de la publicité sur les consciences, Mattelart (1992) en souligne les effets négatifs : «La publicité envahit la vie quotidienne et, mine de rien, elle dicte la norme, détermine la loi; partout elle s'établit de la sorte comme religion totale de ce nouvel âge du libéralisme » (p. 81).

En somme, nous estimons que la scolarisation, même longue, peut mener à une conscience semi-transitive et à l'illusion d'être informé et de pouvoir agir de façon éclairée dans la résolution de problèmes sociaux. C'est particulièrement le cas quand la scolarisation se caractérise par une pédagogie bancaire, quand elle est tributaire de la super-spécialisation et de la fragmentation du savoir, quand elle est mise au service de valeurs marchandes et quand elle ne contrecarre pas les effets nocifs de l'information de masse non critique et de la publicité. La nécessité se fait sentir de susciter un changement en profondeur.

\section{Pour une approche éducative favorisant la conscience transitive}

En nous inspirant de Freire (1973), de Freire (1969) et de Shor (1992), nous postulons que, pour être en mesure de mieux se comprendre et de mieux comprendre autrui et le monde, il faut dépasser la conscience semi-transitive et viser le développement d'une conscience transitive, que nous décririons ainsi :

La conscience transitive est la capacité de réflexion critique qui permet de mettre en doute ce qui est présenté comme inchangeable, de remettre en question ses propres choix ainsi que ceux de la société. C'est la capacité d'accepter d'être dérangé dans sa propre complaisance, de laisser de côté ses propres conceptions "sécurisantes » et de composer avec la complexité, l'ambiguïté, les contradictions, dans la recherche d'une compréhension plus approfondie de la réalité.

Cette capacité permet d'établir des liens plus riches entre ses expériences personnelles et les questions sociales et de constater que la société est une création humaine qu'on peut chercher à comprendre et à transformer, bref, de voir la réalité comme une construction et non comme une fatalité. Il s'agit ici, bien sûr, d'un changement fondamental de paradigme en ce qui concerne le processus menant à la connaissance.

Dans la même ligne de pensée, Shor (1992) et Cummins et Sayers (1995), parmi d'autres, privilégient le recours à un concept apparenté à celui de la conscience transitive, celui de la littératie critique. Ils estiment que la littératie critique est le fruit d'une éducation plus poussée que les formes d'éducation visant à développer d'abord la littératie, laquelle recouvre les habiletés langagières traditionnelles du savoir-lire et du savoir-écrire (Legendre (1993)). Selon Cummins et Sayers (1995), elle va plus loin que la littératie fonctionnelle, qui permet aux personnes de bien 
fonctionner dans les situations sociales et d'emploi typiques de la fin du $\mathrm{XX}^{\mathrm{e}}$ siècle et du début du XXIe siècle dans les pays industrialisés; elle dépasse la littératie culturelle, qui permet de comprendre les textes, les médias et les différentes formes d'interaction sociale à l'intérieur de communautés particulières. La littératie critique est une forme plus complète puisqu'elle permet de décoder les valeurs. Elle comporte, selon Shor (1992) :

les habitudes de la pensée, de la lecture, de l'écriture et de la conversation qui transcendent la signification superficielle, les premières impressions, les mythes dominants, les déclarations officielles, les clichés traditionnels, la sagesse reçue et les simples opinions, afin de comprendre la signification profonde, les causes premières, le contexte social, l'idéologie et les conséquences personnelles d'une action, d'un événement, d'un objet, d'un processus, d'une organisation, d'une expérience, d'un texte, d'une matière d'étude, d'une politique, d'un média de masse ou d'un discours. (p. 129, traduction libre)

Bref, cette conscience transitive, ou littératie critique, est le résultat d'une éducation qui favorise la réflexion critique et l'engagement dans le processus de remise en question de la réalité et des sources d'information.

Le développement de cette conscience transitive est le défi de la PCE que nous analysons dans la deuxième partie du présent article.

\section{En guise de conclusion de cette première partie de l'article}

La société actuelle fait face à une situation très complexe à l'échelle planétaire. Il s'agit d'une situation devant laquelle l'éducation doit œuvrer plus que jamais, estiment un nombre grandissant de spécialistes, au développement de la conscience critique propre à favoriser la compréhension de la complexité de l'être humain et de la réalité sociale ainsi que l'engagement responsable.

Le défi pédagogique demeure celui de transformer les pratiques éducatives à l'école pour qu'elles puissent contribuer, dès l'éducation préscolaire, à l'atteinte des buts visés. Pour y parvenir, il faut, entre autres, que les membres du personnel des écoles soient formés en conséquence, ce qui pose le problème de la formation initiale dans les facultés d'éducation. Si nous prétendons qu'il est nécessaire que les enseignantes et les enseignants deviennent des agents de changement, les facultés d'éducation doivent, elles aussi, transformer leurs missions et leurs pratiques éducatives.

Cet effort visant à repenser la formation initiale en fonction des exigences de la modernité fait partie des grands débats de l'heure dont l'une des questions soulevées est celle de la capacité de l'université de répondre aux exigences complexes de ce monde globalisé qui présente de multiples défis à l'éducation.

C'est le défi qu'a voulu relever la Faculté des sciences de l'éducation de l'Université de Moncton en se donnant une mission et une approche pédagogiques 
qui, tout en respectant la liberté universitaire et la pluralité de paroles, puissent créer un certain consensus chez les membres du corps professoral quant aux valeurs éducatives à promouvoir et les méthodes de travail et de recherche à développer dans le cadre de nos cours, avec l'espoir que les étudiantes et les étudiants soient capables de les mettre en pratique à leur tour dans les écoles.

Cette approche est en devenir et, comme toute démarche pédagogique, elle comporte des limites. Nous invitons les lectrices et les lecteurs à poursuive la réflexion.

Dans cette tentative de repenser la formation initiale en fonction de son rôle d'agent de changement et d'humanisation en ce début du XXI ${ }^{\mathrm{e}}$ siècle, la Faculté s'associe aux forces vives de la société qui sont à l'œuvre dans le monde, à la poursuite d'un idéal de paix, de justice et de respect de la dignité à laquelle a droit tout être humain.

\section{Références bibliographiques}

Allard, Réal (2002). Résistance(s) en milieu francophone minoritaire au Canada. Exploration théorique et analyse du phénomène à partir du vécu langagier et du développement psycholangagier. In Francophonies d'Amérique, Vol. 13, 2002, pp. 7-29.

Audigier, François (2001). Éducation à la citoyenneté dans une perspective planétaire : le cas de la formation initiale et continue du personnel enseignant. In Rencontre des Chaires UNESCO en éducation, Projet des Universités de l'est du Canada, Montréal, 2001.

Audigier, François (2000). Concepts de base et compétences-clés de l'éducation à la citoyenneté démocratique : une deuxième synthèse Document en ligne en cliquant sur ce dernier hyperlien.

Bear-Nicholas, Andrea (1997). Pour franchir le seuil de la paix. In Revue des sciences de l'éducation, Numéro thématique : L'éducation dans une perspective planétaire, sous la direction de Catalina Ferrer, Vol. XXIII(1), 1997, pp. $149-160$.

Bertrand, Yves (1998). Théories contemporaines de l'éducation. Québec : Éditions Nouvelles, 1998.

Bourdieu, Pierre (1999). Donner un sens à l'union. Pour un mouvement social européen. In Le monde diplomatique.

En ligne : http://www.monde-diplomatique.fr/1999/06/BOURDIEU/12158

Breton, Philippe (1997). La parole manipulée. Montréal : Boréal, 1997.

Camps, Victoria (1994). Los valores de la educación. Madrid : Anaya, 1994, 133 p. 
Caouette, Charles (1997). Éduquer. Pour la vie! Montréal : Éditions Écosociété, 1997, 171 p.

CEICI - Centre d'éducation interculturelle et de compréhension internationale (1998). L'éducation à la citoyenneté dans une perspective mondiale. Montréal : CEICI, 1998.

Chomsky, Noam (1996). Les dessous de la politique de l'Oncle Sam. Montréal : Écosociété, 1996, 132 p.

Chomsky, Noam (1995). L'an 501 : La conquête continue. Montréal : Écosociété, 1995, $363 \mathrm{p}$.

Chomsky, Noam (1986). The manufacture of consent. In The Chomsky reader, sous la direction de J. Peck. New York : Pantheon Books, 1986, pp. 121-136.

Chossudovsky, Michel (1998). La mondialisation de la pauvreté : Les conséquences des réformes du FMI et de la Banque mondiale. Montréal : Écosociété, 1998, 248 p.

Chungara, Domitila (1981). Si on me donne la parole. Paris : Maspero.

Commission mondiale sur l'environnement et le développement (1998). Rapport Notre avenir à tous. Montréal : Éditions du Fleuve, 1998.

Cortina, Adela (2001). Cuidadanía económica cosmopolita. In El Pais, Madrid, 5 juin 2001. (En ligne)

Cummins, Jim, Sayers, Dennis (1995). Brave new schools : Challenging cultural illiteracy through global learning networks. Toronto : ON, OISE Press, 1995.

De Beauvoir, Simone (1949). Le deuxième sexe. Paris : Gallimard, 1949.

Duerr, Karlheinz (2000). Stratégies pour apprendre la citoyenneté démocratique. Projet Éducation à la citoyenneté démocratique. Strasbourg : Conseil de l'Europe, 2000, 84 p.

Dumont, René (1998). Un monde intolérable. Le libéralisme en question. Paris : Seuil, 1988.

Ferrer, Catalina (Rédactrice invitée) (1997). Revue des sciences de l'éducation. Numéro thématique : L'éducation dans une perspective planétaire, Vol. XXIII(1), 1997, 232 p.

Ferrer, Catalina (1994). L'éducation pour une perspective mondiale : un défi à relever du niveau préscolaire jusqu'à l'université. In INFO CRDE, Nos 4 et 5 , Moncton, 1994, pp. 1, 4-6.

Forum continental sur l'éducation (2001). Actes du forum continental sur l'éducation, Québec, avril 2001 : L'éducation démocratique, une clé pour le développement des Amériques. Québec : Secrétariat continental sur l'éducation. 
Freire, Paulo (1980). Pédagogie des opprimés. Suivi de Conscientisation et révolution. Paris : Maspero, 1980.

Freire, Paulo (1973). Education for critical consciousness. New York : Seabury, 1973.

Freire, Paulo (1969). La educación como práctica de la libertad. México : Siglo XXI, 1969.

Freund, Andreas (1991). Journalisme et mésinformation. France : La pensée sauvage, 1991, $365 \mathrm{p}$.

Fromm, Erich (1941). La peur de la liberté. Paris : Buchet-Chastel, 1941.

Fromm, Erich (1983). De la désobéissance. Paris : Laffont, 1983, 173 p.

Fromm, Erich (1978). Avoir ou être? Un choix dont dépend l'avenir de l'homme. Paris : Laffont, 1978, 244 p.

FSÉ - Faculté des sciences de l'éducation (1997). Vers une pédagogie actualisante : Mission de la Faculté des sciences de l'éducation et formation initiale à l'enseignement. Moncton, N. B. : Faculté des sciences de l'éducation, Université de Moncton, 1997.

Habachi, René (1986). Fundamentos filosóficos de una universidad para la paz. San José de Costa Rica : Editorial Universidad para la paz, 1986.

Jacquard, Albert (1995). J'accuse l'économie triomphante. Paris : Calmann-Lévy, 1995, 167 p.

Jonas, Hans (1997). Le principe responsabilité : Une éthique pour la civilisation technologique. Paris : Éditions du cerf, 1997, 336 p.

Landry, Rodrigue, Allard, Réal (1997). L'exogamie et le maintien de deux langues et de deux cultures : le rôle de la francité familioscolaire. In Revue des sciences de l'éducation, Vol. XXIII, pp. 561-592.

LeBlanc, Gérard (2001). Allocution d'ouverture. Institut d'été en éducation à la citoyenneté dans une perspective planétaire, Document vidéo. Université de Moncton, Moncton, 2001.

Legendre, Renald (1993). Dictionnaire actuel de l'éducation, (2 éd.). Montréal : Guérin, 1993, 679 p.

Lessard, Claude, Desroches, Fabienne, Ferrer, Catalina (1997). Pour un monde démocratique : l'éducation dans une perspective planétaire. In Revue des sciences de l'éducation, Numéro thématique : L'éducation dans une perspective planétaire, sous la direction de Catalina Ferrer, Vol. XXIII(1), 1997, pp. 3-16.

Magendzo, Abraham, Donoso, Patricio (2000). Cuando a uno lo molestan... : Un acercamiento a la discriminación en la escuela. Santiago de Chile : LOM Ediciones/PIIE, 2000.

Mandela, Nelson (1994). A long walk to freedom. New York : Time Inc, 1994. 
Mayor, Federico (1999). Un monde nouveau. Paris : UNESCO/Ed. Odile Jacob, 1999, 526 p.

Mattelart, Armand, Palmer, M. (1992). Sous la pression publicitaire. In Le monde diplomatique, Manière de voir, 1992, pp. 14-81.

Menchú, Rigoberta (1985). Me llamo Rigoberta Menchú y así me nació la conciencia. In Burgos Elizabeth (1985), México : Siglo veintiuno editores, 1985.

Miller, Allice (1984). C'est pour ton bien : racines de la violence dans l'éducation de l'enfant. Paris : Aubier, 1984.

Morin, Edgar (2000). Les sept savoirs nécessaires à l'éducation du futur. Paris : Seuil, 2000, $130 \mathrm{p}$.

Osorio, Jorge (1999). Educación ciudadana y escuelas para la democracia. In Seminario en educación para la democracia. Santiago de Chile : Ministerio de la educación, 1999.

Osorio, Jorge (1995). La educación para los derechos humanos, su transversalidad e incorporación en los proyectos educativos. In La Piragua, No 11, Santiago de Chile, 1995.

Osorio, Jorge (1988). Polémicas y afirmación de la educación popular en América latina. In La fuerza del arco iris : movimientos sociales, derechos humanos y nuevos paradigmas culturales, sous la direction de Jorge Osorio et Luis Weinstein. Santiago de Chile : CEAAL, 1988, pp. 285-292.

Pagé, Michel, Ouellet, Fernand, Cortesao, Luiza (2001). L'éducation à la citoyenneté Montréal : Éditions du CRP, 2001, 372 p

Perrenoud, Philippe (2000). Fondements de l'éducation scolaire : enjeux de socialisation et de formation.

En ligne : http://www.unige.ch/fapse/SSE/teachers/perrenoud/php_main/ php_2000/2000_24.html

Petrella, Ricardo (2000). Les cinq pièges de l'éducation. Montréal : Fides, 2000.

Reeves, Hubert (1988). Entretien avec Hubert Reeves. In Le devoir, Montréal, 24 mars 1988

Saint-Jean, Armande (1983). Pour en finir avec le patriarcat. Montréal : Éditions Primeur, 1983.

Saramago, José (2000). Entrevue. In El Pais, Madrid, juin 2000. (En ligne)

Sauvé, Lucie (1997). L'approche critique en éducation relative à l'environnement : origines théoriques et applications à la formation des enseignants. In Revue des sciences de l'éducation, Numéro thématique : L'éducation dans une perspective planétaire, sous la direction de Catalina Ferrer, Vol. XXIII(1), 1997, pp. 169-187.

Savater, Fernando (1998). Pour l'éducation. Paris : Payot, 1998, 274 p. 
Semprún, Jorge (1999). Entrevue. In Bouillon de culture, TV5, Paris, 1999.

Shor, Ira (1992). Empowering education : Critical teaching for social change. Chicago : University of Chicago Press, 1992, 286 p.

Statistique Canada (1998). Enquête internationale sur l'alphabétisation des adultes : le Nouveau-Brunswick en un clin d'œeil. Ottawa, auteur, 1998, 60 p.

Taylor, Charles (1992). Grandeur et misère de la modernité. Québec : Bellarmin, 1992, $150 \mathrm{p}$.

Thériault, Joseph-Yvon (1999). Francophonies minoritaires au Canada : l'état des lieux. Moncton, NB : Éditions d'Acadie.

Touraine, Alain (1997). Pourrons-nous vivre ensemble? Égaux et différents. Paris : Fangard, 1997.

Touraine, Alain (2001). Entrevue. In El Pais, Madrid, 14 juillet 2001. (En ligne)

UNESCO (2000). Rapport mondial sur l'éducation 2000. Le droit à l'éducation. Vers l'éducation pour tous, tout au long de la vie. Paris : Éditions UNESCO, 2000, $182 \mathrm{p}$.

UNESCO (1993). La tolérance aujourd'hui. Paris : UNESCO.

UNESCO (1984). L'éducation pour la coopération internationale et la paix dans l'enseignement primaire. Paris : Éditions UNESCO, 1984.

UNESCO (1945). Acte constitutif. Paris : Éditions UNESCO, 1945.

UNICEF (2000). La situation des enfants dans le monde. Genève : UNICEF, 2000.

Vienneau, Raymond (2001). Besoins de formation et pratiques pédagogiques en éducation à la citoyenneté dans une perspective planétaire. In Nouvelles, No 6, février, No 7, mars 2001.

Weinstein, Luis (1988). La conciencia humanista y los Derechos Humanos. In La fuerza del arco iris : movimientos sociales, derechos humanos y nuevos paradigmas culturales, Sous la direction de Jorge Osorio et Luis Weinstein. Santiago de Chile : CEAAL, 1988, pp. 21-36.

Wurman, Richard Saul (1989). Information anxiety. New York : Doubleday, 1989. Ziegler, Jean (2001). Entrevue. In El mundo, Madrid, 16 octobre 2001. (En ligne) 